\title{
Too bored to bother? Boredom as a potential threat to the efficacy of pandemic containment measures
}

Corinna S. Martarelli (iD ${ }^{1,4 凶} \&$ Wanja Wolff $2,3,4$

Coronavirus disease 2019 (COVID-19) caused by coronavirus (SARS-nCoV2) is currently spreading across the world. In response, different sets of pandemic containment measures have been employed by several countries. The effectiveness of non-pharmacological measures such as home confinement hinges on adherence by the population. While adherence to these social distancing measures appears to be high in general, adherence might be more challenging for some individuals and complying with these measures might become more difficult the longer they last. Here, we suggest that boredom and self-control are two important psychological concepts for understanding the challenges the COVID-19 pandemic containment measures pose to individuals. To maximize adherence to these measures, we propose to consider the specific and combined effects of boredom and self-control demands elicited by this situation on subsequent behavior.

\footnotetext{
${ }^{1}$ Faculty of Psychology, Swiss Distance University Institute, Brig, Switzerland. ${ }^{2}$ Department of Sport Science, University of Konstanz, Konstanz, Germany. ${ }^{3}$ Department of Educational Psychology, University of Bern, Bern, Switzerland. ${ }^{4}$ These authors contributed equally: Corinna S. Martarelli, Wanja Wolff.

凶email: corinna.martarelli@fernuni.ch
} 


\section{Current situation}

he worldwide spread of Coronavirus disease 2019 (COVID-19) has been called a pandemic by the World Health Organization (WHO, 2020). COVID-19 is highly infectious and it can have severe health consequences and even lead to death (Huang et al., 2020). Currently (May 2020), there are no therapeutic agents available and there is presumably no pre-existing immunity in the population against COVID-19. As COVID-19 is spreading exponentially around the world, there has been an urgent call for targeted action (European Center for Disease Prevention and Control, 2020). Given the current situation, several non-pharmacological pandemic containment measures have been employed by many countries. These measures include isolation, quarantine, ${ }^{1}$ social distancing (sometimes also referred to as physical distancing), suspension of gatherings, closing of borders, schools, and workplaces among others. Moreover, several countries have adopted nationwide home confinement periods, during which everyone must stay at home, with no physical contact with other persons not living in the same home.

\section{Human behavior during a pandemic}

Measures like home confinement can only be effective if the public adheres to them. At the time of writing, general compliance appears to be high in the US (Wolff et al., 2020), although compliance rates appear to somewhat differ among countries (Travaglino and Moon, 2020). However, gatherings of people have still been observed in different countries even though full home confinement has been ordered (Valiante, 2020). In some countries, people have even gathered to protest the COVID-19 pandemic containment measures (Deutsche Welle, 2020), although social distancing has been regarded as the currently most effective way to slow the spread of COVID-19 (Gollwitzer et al., 2020). So, the question arises why do some people not stay at home even though these measures are imposed to slow the spreading of the COVID-19 pandemic? Staying at home comes with costs, such as reduced social and physical contact, loss of freedom, loss of routines, and a recent review (Brooks et al., 2020) confirmed the aversive psychological impact of confinement measures, especially in terms of anxiety and depressive symptoms (see also Park and Park, 2020). The authors (Brooks et al., 2020) identified several stressors during home confinement, including duration of confinement, fears of infection, inadequate supplies, inadequate information, and boredom. Even though at first glance it might be appealing to have time to do nothing, research shows that being left alone with one's own thoughts might cause boredom, and boredom is perceived as highly aversive: in one study, some participants even preferred to self-administer mild electric shocks instead of being on their own with their thoughts (Wilson et al., 2014).

Here, we draw upon recent theorizing on the potential interplay between boredom and self-control in guiding goal-directed behavior (Wolff and Martarelli, 2020) to emphasize the psychological challenges pandemic containment measures impose on the individual. We develop the hypothesis that both sensations of boredom and perceived self-control demands likely affect the efficacy of pandemic containment measures (see Fig. 1 for the proposed working model). Both concepts can contribute to understand why people find it difficult to stay at home even though these measures have personal and public health benefits. Our goal is to highlight how boredom (subsection "Boredom as a signal to change behavior"), self-control (subsection "Exerting self-control reduces willingness to invest effort"), and their interplay (subsection "The combined challenge of boredom and self-control") are likely to affect compliance with the current pandemic containment measures and to stimulate thinking about possibilities to enhance individual adherence to these measures (section "Concluding remarks").

Boredom as a signal to change behavior. Boredom is a psychological state that most people experience on a regular basis (Harris, 2000). The Meaning and Attentional Components (MAC) model of boredom proposes that attentional failure (i.e., being unable to successfully engage attention with an activity) and/or a perceived lack of meaning can lead to boredom (Westgate and Wilson, 2018). Most prototypically, boredom occurs when stimulation is low. As quotes related to home confinement reveal, ${ }^{2}$ boredom can be a challenge to confined people. Boredom might be exacerbated when an activity/situation is perceived as meaningless. This might be the case, if one does not believe in the efficacy and/or necessity of being in home confinement for slowing the spread of COVID-19.

Being bored affects behavior. High-boredom proneness (trait boredom) has been linked to various negative outcomes (Eastwood et al., 2012), like gambling behavior (Goldstein et al., 2016), poor mental health (Binnema, 2004), violent offenses (Dåderman and Lidberg, 1999), and even youth suicide (Heled and Read, 2005). However, other findings indicate that boredom can also trigger pro-social interactions (Van Tilburg and Igou, 2017) or creativity (Harris, 2000). In light of its' high prevalence in everyday life and its' powerful effect on behavior, researchers have become increasingly interested in the specific function boredom might have in guiding behavior (Geana et al., 2016; GomezRamirez and Costa, 2017). For example, recent theorizing proposes that boredom's primary function is to instigate a change in behavior (Wolff and Martarelli, 2020). The mechanisms by which boredom is thought to instigate this change is via a discounting of an ongoing activity's current value and an increased orientation towards more rewarding behavioral alternatives. Thus, a person that is adhering to the pandemic containment measures, might get bored because the available behavioral options become less attractive as a function of exposure, while attention for more rewarding alternatives increases as a function of time. For example, watching TV while in home confinement is likely to become less attractive over time, while the joy of going out with friends will become ever more salient. Further, when one's current activity diminishes in value, other options are likely to increase in relative value, prompting people to engage in (potentially detrimental) activities they would normally abstain from. Thus, boredom should make adherence to containment measures more difficult.

Importantly, people differ in their boredom proneness. Specifically, research on trait boredom indicates that compared to females, males have a higher tendency to get bored (Vodanovich and Kass, 1990), whereas age is negatively associated with boredom proneness (Isacescu et al., 2017). This suggests that boredom-induced challenges to adhering to pandemic containment measures are likely to vary between individuals. Indeed, preliminary research regarding compliance with COVID-19 containment measures in China indicates that males where more likely to visit crowed places and less likely to wear a mask (Zhong et al., 2020). Taken together, (some) COVID-19 containment measures are likely to induce boredom, and this might make adhering to these measures particularly challenging for individuals that are high in boredom proneness. Indeed, a recent study provides tentative support for this proposition: high-boredom proneness was associated with lower self-reported adherence to social distancing and a higher likelihood of having contracted COVID-19 (Wolff et al., 2020). Importantly, the association between boredom and adherence was mediated by perceptions of difficulty. Thus, individuals with 


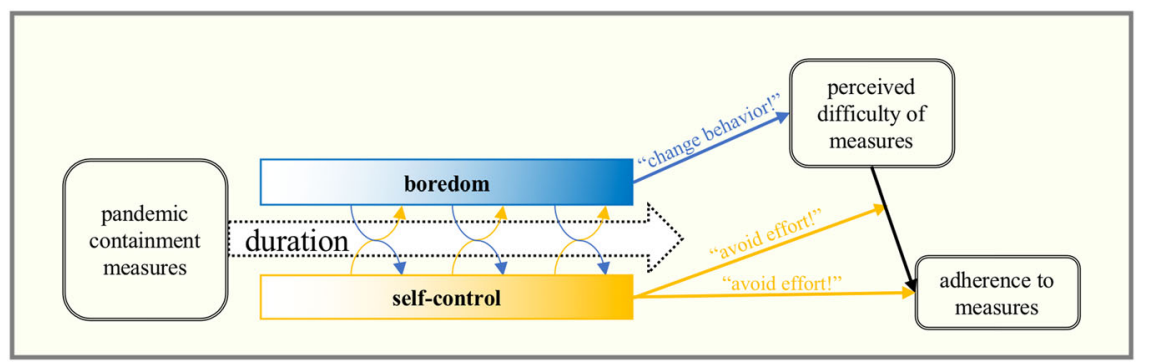

Fig. 1 Simplified adaptation of the theoretical model proposed by Wolff and Martarelli (2020) on the potential interplay between boredom and selfcontrol during the current COVID-19 containment measures. The model proposes that boredom and self-control demands change over time (duration arrow in the model), changes illustrated by the blue and yellow gradients highlighting increases in boredom and self-control demands during pandemic containment measures. Please note that changes must not be linear, the figure is schematic and meant to illustrate the dynamical interplay between boredom and self-control. According to the model, boredom signals whether one should explore behavioral alternatives ("change behavior!" arrow in the model) and self-control demands signal whether one should stop investing further effort ("avoid effort!" arrows in the model). We propose that adhering to the pandemic containment measures leads to more boredom in boredom prone individuals, thereby making adherence more difficult for them. Thus, the effect boredom has on adherence should be mediated by the perceived difficulties to adhere to these measures (mediation hypothesis). Adhering to the measures, although it is difficult, should rely on self-control. Therefore, the model proposes that high-trait self-control buffers the effect of difficulties on adherence (moderation hypothesis). A recent study has provided first empirical evidence for the proposed mechanisms (Wolff et al., 2020). Crucially, there are other variables that are likely to influence the perceived difficulty of the measures and the adherence to the measures. For example, variables such as fear of the virus (Harper et al., 2020), understanding of the risk posed by the virus (Pennycook et al., 2020), or socio-cultural context (e.g., inequalities in access to resources, economic disadvantage, racial discrimination, culture, ideological differences; Van Bavel et al., 2020) also affect adherence to pandemic containment measures. Research is needed to unravel how these variables covary with the experience of boredom and the exertion of self-control (e.g., different socio-cultural contexts might be linked with different capacities to be bored).

high-boredom proneness perceived adherence to social distancing as being more difficult and were consequently less likely to practice social distancing.

Exerting self-control reduces willingness to invest effort. Selfcontrol, loosely defined as the capacity to control predominant behavioral impulses (Ridder et al., 2012), is strongly related with a plethora of positive outcomes (Moffitt et al., 2011). It is conceivable that-in addition to inducing boredom-COVID-19 containment measures place substantial self-control demands on the individual. For example, self-control is required to control the habitual response of shaking hands with friends (incidentally, the voluntary control of such habitual actions has been used in selfcontrol trainings because of the demands for self-control they impose; Friese et al., 2017).

However, applying control is perceived as effortful and aversive (Wolff et al., 2019). Consequently, people try to avoid the exertion of control (Kurzban et al., 2013). More specifically, if two activities yield the same reward, people will generally choose the one that demands less self-control. ${ }^{3}$ Thus, self-control is only applied when its' benefits outweigh its' costs. Accordingly, recent theorizing on self-control proposes that the sensation of effort that accompanies the exertion of self-control has the function of signaling the costs of control (Kurzban et al., 2013). Exerting selfcontrol affects subsequent acts of self-control on the behavioral (Hagger et al., 2010) and/or the perceptual level (Milyavskaya et al., 2019). More precisely, a large body of research indicates that exerting self-control leads to impaired performance in subsequent activities that rely on self-control (Cunningham and Baumeister, 2016). While the existence and magnitude of these behavioral effects is debated (Carter and McCullough, 2014; Wolff et al., 2018), a relatively consistent body of research shows that self-control exertion leads to feelings of fatigue and frustration (Wolff et al., 2019). The latter finding is consistent with theoretical accounts proposing that these sensations have the function of tracking the costs of control (Shenhav et al., 2017). This implies that rising costs of control will skew the cost-benefit analysis and make control application unfavorable. Specifically, recent functional theorizing on self-control proposes that applying control reduces the willingness to invest further effort (Wolff and Martarelli, 2020). For example, during the first day of home confinement one might be more willing to apply the necessary self-control that is required to comply with the measures than after 1 full week. Providing tentative support for the ideas presented here, a recent study showed that low-trait selfcontrol was associated with lower adherence to social distancing (Wolff et al., 2020). Importantly, self-control also moderated the relationship between perceived difficulties to adhere and actual adherence. Thus, individuals with low-trait self-control were less able or willing to apply the effort needed to adhere to social distancing when this was perceived as difficult.

The combined challenge of boredom and self-control. Crucially, it has been proposed that the sensation of boredom and the perceived costs of self-control exertion uniquely and interdependently affect behavior (Wolff and Martarelli, 2020): While boredom signals that one should do something else, the exertion of self-control signals that one should avoid further exertion of effort. If the current situation requires to sustain boredom (e.g., due to a lack of behavioral alternatives), then resisting the urge to do something else relies on self-control. However, if compliance with containment measures has already relied on self-control, then this is likely to reduce the willingness to ward off the boredom-induced behavioral impulses. Similarly, in the beginning, some of the self-control demands that are created by the containment measures might be somewhat motivating. For example, resisting the tendency to touch doorknobs or other potentially dangerous surfaces might pose a playful challenge that one can try to excel at. However, after having done this for a prolonged time, the not-touching of dangerous surfaces will not be a challenge anymore, but it might become boring.

Finally, research indicates that boredom proneness and selfcontrol are inversely related on the trait level (Mugon et al., 2018). Thus, people, who tend to get bored also tend to display low selfcontrol. In regard to the COVID-19 pandemic, this is important: the structural properties of the necessary containment measures 
are likely to induce boredom and pose self-control demands. These challenges are likely to become stronger, the longer the containment measures last. Trait differences in the tendency to be vulnerable to these challenges are likely to covary in a systematic fashion. Thus, it is likely that a person who gets bored quickly during home confinement also has less self-control to control boredom-induced behavioral impulses. Preliminary evidence indicates that younger (male) adults show lowest compliance with containment measures (Zhong et al., 2020). Incidentally, research indicates that boredom proneness tends to be high (Isacescu et al., 2017) and the negative consequences of low selfcontrol appear to be more pronounced (Ridder et al., 2012) in this group. Given that young adults appear to be the most likely carriers of the SARS-CoV-2 virus, it is crucial to turn to the psychological demands that potentially make compliance to confinement regulations particularly challenging to this group of people. Interventions could be tailored specifically to combat boredom and reduce self-control demands in this potentially critical subpopulation.

\section{Concluding remarks}

Pandemic containment measures have been shown to be associated with aversive experiences, including boredom (Brooks et al., 2020) and adherence despite being bored is likely to be selfcontrol demanding (Wolff and Martarelli, 2020). Since boredom is understood to be a powerful motivator for behavioral change (Westgate and Wilson, 2018), it might lead to negative (e.g., ignoring social distancing recommendations) or positive (e.g., volunteering to do grocery shopping for the elderly) behavioral impulses. Consequently, interventions that highlight positive behavioral alternatives might be a cost-effective way to channel this impulse in a manner that is conducive to personal and public health. On an individual level, learning to recognize and use boredom in an adaptive manner might be useful. For example, making a list of realistic alternatives that are perceived as interesting and engaging, to be used when boredom arises might be promising. Next, to create optimal conditions in the environment, other possibilities to improve adhesion to containment measures are more related to internal changes, such as refocusing on the meaning of pandemic containment measures (i.e., public health) when boredom arises. This strategy should in turn also make adhering to containment measures less self-control demanding. Others before us have proposed that knowing that people benefit from one's home confinement make it easier to adhere to home confinement (Brooks et al., 2020). The importance of meaning is highlighted by recent theorizing on boredom (Westgate and Wilson, 2018) showing that perceived lack of meaning is associated with the experience of boredom.

As countries begin to gradually relax some of the pandemic containment measures, it is important to think about the effect this might have on boredom and self-control. While it is conceivable that boredom goes down, self-control demands might actually increase during the process of easing. Although the expected alleviation of boredom might reduce some of the selfcontrol demands, an easing of restrictions is also likely to add new challenges to self-control. Having more individual responsibility in regard to one's COVID-19-related behavioral choices might increase self-control demands because these choices can be difficult to make. Indeed, research shows that having to make choices can be self-control demanding in its' own right (Vohs et al., 2008). Moreover, several measures, such as keeping a specified distance at all time or hand sanitizing will continue to place substantial self-control demands on the individuals. Especially, when aspects of the social environment make it difficult to adhere to these measures. Thus, if home confinement was experienced as effortful, individuals might simply not be willing to further exert the required effort. Therefore, interventions that are tailored to lower the situational self-control demands might help individuals that struggle with the easing of restrictions.

To conclude, boredoms motivational force poses a risk as well as an opportunity during the current COVID-19 pandemic. A currently highly popular meme states "Your grandparents were asked to go to war. All you are being asked to do is sit on the couch. You can do this" (https://makeameme.org/meme/yourgrandparents-were, 2020). Clearly, going to war is not comparable in terms of difficulty to sitting on the couch. In the current paper, we focused on home confinement measures and suggested that the interplay of boredom (signaling to change behavior) and selfcontrol demands (signaling to avoid effort) make the task of sitting on the couch more challenging than it might appear at first glance.

Received: 24 March 2020; Accepted: 10 June 2020;

Published online: 09 July 2020

\section{Notes}

1 Isolation is the separation of individuals who have been diagnosed with COVID-19, whereas quarantine is the separation of individuals who have potentially been exposed to COVID-19 (Centers for Disease Control and Prevention, 2017).

2 As for example, "time flies-that's the saying. However, in this situation, it can go slow. The days are long” (Ribeiro, 2020).

3 However, it has recently been suggested that the opposite can also be true: exerting self-control across time can also have its own value and individuals sometimes choose alternatives because they require more effort (Inzlicht et al., 2018).

\section{References}

Binnema D (2004) Interrelations of psychiatric patient experiences of boredom and mental health. Issues Ment Health Nurs 25:833-842. https://doi.org/10.1080/ 01612840490506400

Brooks SK, Webster RK, Smith LE et al. (2020) The psychological impact of quarantine and how to reduce it: rapid review of the evidence. The Lancet 395:912-920. https://doi.org/10.1016/S0140-6736(20)30460-8

Carter EC, McCullough ME (2014) Publication bias and the limited strength model of self-control: has the evidence for ego depletion been overestimated? Front Psychol 5:823. https://doi.org/10.3389/fpsyg.2014.00823

Centers for Disease Control and Prevention (2017) Quarantine and isolation. Centers for Disease Control and Prevention. https://www.cdc.gov/ quarantine/index.html

Cunningham MR, Baumeister RF (2016) How to make nothing out of something: analyses of the impact of study sampling and statistical interpretation in misleading meta-analytic conclusions. Front Psychol 7:1639. https://doi.org/ 10.3389/fpsyg.2016.01639

Dåderman AM, Lidberg L (1999) Flunitrazepam (Rohypnol) abuse in combination with alcohol causes premeditated, grievous violence in male juvenile offenders. J Am Acad Psychiatry Law 27:83-99

Deutsche Welle (2020) https://www.dw.com/en/coronavirus-latest-italy-outlinesloosening-of-lockdown/a-53460404

Eastwood JD, Frischen A, Fenske MJ, Smilek D (2012) The unengaged mind: defining boredom in terms of attention. Perspect Psychol Sci 7:482-495. https://doi.org/10.1177/1745691612456044

European Centre for Disease Prevention and Control (2020) Rapid risk assessment: novel Coronavirus disease 2019 (COVID-19) pandemic: increased transmission in the EU/EEA and the UK-sixth update. European Centre for Disease Prevention and Control. https://www.ecdc.europa.eu/en/publications-data/ rapid-risk-assessment-novel-coronavirus-disease-2019-covid-19-pandemicincreased

Friese M, Frankenbach J, Job V, Loschelder DD (2017) Does self-control training improve self-control? A meta-analysis. Perspect Psychol Sci 12:1077-1099. https://doi.org/10.1177/1745691617697076

Geana A, Wilson R, Daw ND, Cohen J (2016) Boredom, information-seeking and exploration. In: Papafragou A, Grodner D, Mirman D, Trueswell JC (eds) Proceedings of the annual conference of the Cognitive Science Society, Cognitive Science Society, Austin, TX, pp. 1751-1756

Goldstein AL, Vilhena-Churchill N, Stewart SH et al. (2016) Mood, motives, and money: an examination of factors that differentiate online and non-online young adult gamblers. J Behav Addict 5:68-76. https://doi.org/10.1556/ 2006.5.2016.003 
Gollwitzer A, Martel C, Marshall J, Höhs JM, Bargh JA (2020) Connecting selfreported social distancing to real-world behavior at the individual and U.S. state level. https://doi.org/10.31234/osf.io/kvnwp

Gomez-Ramirez J, Costa T (2017) Boredom begets creativity: a solution to the exploitation-exploration trade-off in predictive coding. Biosystems 162:168-176. https://doi.org/10.1016/j.biosystems.2017.04.006

Hagger MS, Wood C, Stiff C, Chatzisarantis NLD (2010) Ego depletion and the strength model of self-control: a meta-analysis. Psychol Bull 136:495-525. https://doi.org/10.1037/a0019486

Harper CA, Satchell L, Fido D, Latzman R (2020) Functional fear predicts public health compliance in the COVID-19 pandemic. Int J Ment Health Addict https://doi.org/10.1007/s11469-020-00281-5

Harris MB (2000) Correlates and characteristics of boredom proneness and boredom. J Appl Soc Psychol 30:576-598. https://doi.org/10.1111/j.15591816.2000.tb02497.x

Heled E, Read J (2005) Young peoples' opinions about the causes of, and solutions to, New Zealand's high youth suicide rate. Suicide Life Threat Behav 35:170-180. https://doi.org/10.1521/suli.35.2.170.62881

Huang C, Wang Y, Li X et al. (2020) Clinical features of patients infected with 2019 novel coronavirus in Wuhan. The Lancet 395:497-506. https://doi.org/ 10.1016/S0140-6736(20)30183-5

Inzlicht M, Shenhav A, Olivola CY (2018) The effort paradox: effort is both costly and valued. Trends Cogn Sci 22:337-349. https://doi.org/10.1016/j.tics.2018.01.007

Isacescu J, Struk AA, Danckert J (2017) Cognitive and affective predictors of boredom proneness. Cogn Emot 31:1741-1748. https://doi.org/10.1080/ 02699931.2016 .1259995

Kurzban R, Duckworth A, Kable JW, Myers J (2013) An opportunity cost model of subjective effort and task performance. Behav Brain Sci 36:6. https://doi.org/ 10.1017/S0140525X12003196

Milyavskaya M, Inzlicht M, Johnson T, Larson MJ (2019) Reward sensitivity following boredom and cognitive effort: a high-powered neurophysiological investigation. Neuropsychologia 123:159-168. https://doi.org/10.1016/j. neuropsychologia.2018.03.033

Moffitt TE, Arseneault L, Belsky D et al. (2011) A gradient of childhood selfcontrol predicts health, wealth, and public safety. Proc Natl Acad Sci USA 108:2693-2698. https://doi.org/10.1073/pnas.1010076108

Mugon J, Struk A, Danckert J (2018) A failure to launch: regulatory modes and boredom proneness. Front Psychol 9:1126. https://doi.org/10.3389/fpsyg.2018.01126

Park S-C, Park YC (2020) Mental health care measures in response to the 2019 novel coronavirus outbreak in Korea. Psychiatry Investig 17:85-86. https:// doi.org/10.30773/pi.2020.0058

Pennycook G, McPhetres J, Bago B, Rand DG (2020) Predictors of attitudes and misperceptions about COVID-19 in Canada, the U.K., and the U.S.A. https:// doi.org/10.31234/osf.io/zhjkp

Ribeiro C (2020) The family lockdown guide: how to emotionally prepare for coronavirus quarantine. The Guardian. https://www.theguardian.com/world/ 2020/mar/13/the-family-lockdown-guide-how-to-emotionally-prepare-forcoronavirus-quarantine

Ridder DTD, Lensvelt-Mulders G, Finkenauer C et al. (2012) Taking stock of self-control: a meta-analysis of how trait self-control relates to a wide range of behaviors. Personal Soc Psychol Rev 16:76-99. https://doi.org/10.1177/1088868311418749

Shenhav A, Musslick S, Lieder F et al. (2017) Toward a rational and mechanistic account of mental effort. Annu Rev Neurosci 40:99-124. https://doi.org/ 10.1146/annurev-neuro-072116-031526

Travaglino GA, Moon C (2020) Explaining compliance with social distancing norms during the COVID-19 pandemic: the roles of cultural orientations, trust and self-conscious emotions in the US, Italy, and South Korea. https:// doi.org/10.31234/osf.io/8yn5b

Valiante G (2020) COVID-19: governments threaten fines, arrest, if people refuse to follow orders to isolate and social distance. Canadian Press. https:// nationalpost.com/news/governments-threaten-fines-arrest-if-orders-todistance-and-isolate-not-followed

Van Bavel JJ, Baicker K, Boggio PS et al. (2020) Using social and behavioural science to support COVID-19 pandemic response. Nat Hum Behav. https:// doi.org/10.1038/s41562-020-0884-z
Van Tilburg WAP, Igou ER (2017) Boredom begs to differ: differentiation from other negative emotions. Emotion 17:309-322. https://doi.org/10.1037/ emo0000233

Vodanovich SJ, Kass SJ (1990) Age and gender differences in boredom proneness. J Soc Behav Pers 5:297-307

Vohs KD, Baumeister RF, Schmeichel BJ, Twenge JM, Nelson NM, Tice DM (2008) Making choices impairs subsequent self-control: a limited-resource account of decision making, self-regulation, and active initiative. Motiv Sci 1:19-42. https://doi.org/10.1037/2333-8113.1.S.19

Westgate EC, Wilson TD (2018) Boring thoughts and bored minds: the MAC model of boredom and cognitive engagement. Psychol Rev 125:689-713. https://doi.org/10.1037/rev0000097

WHO (2020) WHO announces COVID-19 outbreak a pandemic. http://www.euro. who.int/en/health-topics/health-emergencies/coronavirus-covid-19/news/ news/2020/3/who-announces-covid-19-outbreak-a-pandemic

Wilson TD, Reinhard DA, Westgate EC et al. (2014) Just think: the challenges of the disengaged mind. Science 345:75-77. https://doi.org/10.1126/science. 1250830

Wolff W, Baumann L, Englert C (2018) Self-reports from behind the scenes: questionable research practices and rates of replication in ego depletion research. PLoS ONE 13:0199554. https://doi.org/10.1371/journal.pone.0199554

Wolff W, Martarelli CS (2020) Bored into depletion? Towards a tentative integration of perceived self-control exertion and boredom as guiding signals for goal-directed behavior. Perspect Psychol Sci. https://doi.org/10.1177/ 1745691620921394

Wolff W, Martarelli CS, Schüler J, Bieleke M (2020) High boredom proneness and low trait self-control impair adherence to social distancing guidelines during the COVID-19 pandemic. https://doi.org/10.31234/osf.io/jcf95

Wolff W, Sieber V, Bieleke M, Englert C (2019) Task duration and task order do not matter: no effect on self-control performance. Psychol Res 1-11. https:// doi.org/10.1007/s00426-019-01230-1

Zhong BL, Luo WW, Li HM et al. (2020) Knowledge, attitudes, and practices towards COVID-19 among Chinese residents during the rapid rise period of the COVID-19 outbreak: a quick online cross-sectional survey. Int J Biol Sci 16:1745-1752. https://doi.org/10.7150/ijbs.45221

\section{Competing interests}

The authors declare no competing interests.

\section{Additional information}

Correspondence and requests for materials should be addressed to C.S.M.

Reprints and permission information is available at http://www.nature.com/reprints

Publisher's note Springer Nature remains neutral with regard to jurisdictional claims in published maps and institutional affiliations.

Open Access This article is licensed under a Creative Commons Attribution 4.0 International License, which permits use, sharing, adaptation, distribution and reproduction in any medium or format, as long as you give appropriate credit to the original author(s) and the source, provide a link to the Creative Commons license, and indicate if changes were made. The images or other third party material in this article are included in the article's Creative Commons license, unless indicated otherwise in a credit line to the material. If material is not included in the article's Creative Commons license and your intended use is not permitted by statutory regulation or exceeds the permitted use, you will need to obtain permission directly from the copyright holder. To view a copy of this license, visit http://creativecommons.org/ licenses/by/4.0/

(C) The Author(s) 2020 